IMSc $/ 2003 / 05 / 10$

\title{
The Landau electron problem on a cylinder
}

\author{
G. Date* \\ The Institute of Mathematical Sciences \\ CIT Campus, Chennai-600 113, INDIA. \\ P. P. Divakaran \\ The Institute of Mathematical Sciences \\ CIT Campus, Chennai-600 113, INDIA. and \\ Chennai Mathematical Institute \\ 92, G. N. Chetty Road, T. Nagar, \\ Chennai-600 017, INDIA.
}

\begin{abstract}
We consider the quantum mechanics of an electron confined to move on an infinite cylinder in the presence of a uniform radial magnetic field. This problem is in certain ways very similar to the corresponding problem on the infinite plane. Unlike the plane however, the group of symmetries of the magnetic field, namely, rotations about the axis and the axial translations, is not realized by the quantum electron but only a subgroup comprising rotations and discrete translations along the axial direction, is. The basic step size of discrete translations is such that the flux through the 'unit cylinder cell' is quantized in units of the flux quantum. The result is derived in two different ways: using the condition of projective realization of symmetry groups and using the more familiar approach of determining the symmetries of a given Hamiltonian.

PACS numbers: 02.20, 03.65.-w
\end{abstract}

*Electronic address: shyam@imsc.res.in

${ }^{\dagger}$ Electronic address: ppd@imsc.res.in 


\section{INTRODUCTION}

The classic 'Landau electron' problem deals with the quantum mechanics of an electron on an infinite plane with a uniform magnetic field perpendicular to the plane. It has been thoroughly studied for decades and is the basis for many experimental investigations notably in the context of the Hall effect. Apart from its practical relevance, it also exhibits many theoretically interesting features. It is exactly solvable with a simple spectrum for the Hamiltonian; it has infinite degeneracy for all energy eigenvalues; this degeneracy is organized in an irreducible representation of a Heisenberg group and so on. Interestingly the Heisenberg group arises as the central extension of the translation subgroup of the symmetry group of the plane by the $U(1)$ group of phases, the central extension being determined by the magnetic field. This is also a dramatic illustration of the Wigner theorem 1] namely, that symmetries are realized projectively in quantum mechanics i.e. the quantum state space carries a projective unitary representation (PUR) of a symmetry group. It is dramatic in the sense that a free particle on the plane realizes the translation symmetry by a unitary representation (UR) but if it is charged, the presence of a uniform magnetic field forces a non-trivial projective realization.

In this work we consider a variant of the Landau electron problem by replacing the infinite plane by an infinite cylinder. The magnetic field is again uniform and perpendicular to the surface of the cylinder, i.e. is radially directed and constant in magnitude. This is again exactly solvable and displays the classic features of the planar case but with an interesting twist. The invariance of the magnetic field under continuous translations along the axial direction cannot be realized by the quantum electron. However, a discrete subgroup is realized as a symmetry. The basic reason for this is that there are simply no non-trivial, continuous, projective, unitary representations of the cylinder group, $S O(2) \times \mathbb{R}$. Its subgroup $S O(2) \times \mathbb{Z}$ however does have such (infinite dimensional) representations with properties analogous to the planar case. The step size of the discrete translation is controlled by the magnetic field so as to make the flux through the 'unit cylinder cell' an integral multiple of the flux quantum.

The surprising nature of the result prompted us to reanalyze the problem using the usual methods based on a Hamiltonian involving the gauge potential explicitly and looking for its symmetries. The same result, including the condition on the step size, is obtained again. The mechanism is a subtle interplay of gauge invariance and the definition of symmetry in 
the context of external gauge potentials. The non-contractible nature of the cylinder implies that there is a 1-parameter family of gauge-equivalence classes, all giving the same magnetic field. Within each class, only the discrete subgroup of the cylinder group acts as a symmetry group.

We would like to clarify at the outset our approach. It is well known that when the configuration space of a system is topologically non-trivial (non-contractible), there is no unique quantization procedure for that classical system [2]. Many of these are usually in the canonical approach based on the classical phase space. In this paper however our approach is somewhat different.

We identify a group of symmetries of the configuration space which we expect to be realized in the state space whatever be the quantization procedure adopted. The symmetry realization must be via a PUR (equivalently by a UR of a suitable central extension) thanks to Wigner's theorem. If the centrally extended group happens to be a 'Heisenberg group' i.e. has a unique (up to unitary equivalence) unitary, irreducible representation, then different quantizations can at most differ in the multiplicities (reflected in the degeneracies of the energy spectrum).

It will turn out that the expected symmetry group of the configuration space (the cylinder), namely the cylinder group has no continuous PURs and thus there cannot be any quantization which will realize the symmetry. However, we will find that its subgroup, $S O(2) \times \mathbb{Z}$, can be admitted as the symmetry group and exhibit a quantum state space which does so together with the dynamics.

The important physical implication is that in this system the electron will have only the reduced symmetry of discrete axial translations.

The paper is organized is follows.

In section II, we present the analysis based on the PUR's of symmetry groups. This is divided in five subsections. After describing the symmetry based approach we recall some basic facts from projective unitary representations of Abelian groups and their relation to the unitary representations of their central extensions by $U(1)$ in subsection A. The approach is illustrated for the familiar planar Landau electron problem, in subsection $\mathrm{B}$. The nonexistence of PUR's of the cylinder group is discussed next in subsection C. In subsection D, the PUR's of the subgroup of the cylinder group, appropriate for discrete axial translations, are obtained. In subsection E, the wave function representation together with the projective 
action are detailed. The form of the invariant Hamiltonian is also derived here.

In section III, we carry out the analysis beginning with the Hamiltonian of the system. This is divided in four subsections. In subsection A, we note the key points which lead to the result and specify the class of wavefunctions and the (gauge dependent) Hamiltonian. In subsection $\mathrm{B}$, we specify the gauge transformation, parameterize the gauge potentials giving the same magnetic field and classify them into gauge equivalence classes using the notion of holonomy of gauge potentials. This is followed by the definition of symmetry transformations in this context in subsection C. Here, the classification of gauge potentials is used to deduce that only transformations corresponding to the discrete subgroup of the cylinder group qualify to be termed symmetry transformations. The apparent reduction of symmetry is manifested at this stage itself. In the last subsection D, the spectrum of the Hamiltonian and unitary operators implementing the symmetries are presented and shown to reproduce the results from section II.

Section IV contains concluding remarks.

\section{THE SYMMETRY APPROACH}

The approach taken in this section can be described as constructing a quantum system as realizing certain natural symmetry groups. The approach begins by specifying a configuration space for a given system and choosing a group, $G$, of transformations of the configuration space as the group of symmetries of the system. Quite generally, symmetry groups are realized in a quantum framework as projective, unitary representations (PUR), unitary representations being a special (trivial) case of these. This follows from the superposition principle via the Wigner theorem [1] on symmetries. Mathematically, such projective representations can be obtained as ordinary unitary representations of suitable central extensions $\tilde{G}$ of $G$. The statement that $G$ is a symmetry group of the system entails the existence of a family of Hamiltonians, one for each equivalence class of central extensions, and invariant under the action of that particular extension [3] .

For example, let the physical system be a charged particle moving in a plane in the presence of a uniform magnetic field of strength $B$, perpendicular to the plane. The obvious symmetries of the system form the Euclidean group, $E_{2}$, the semi-direct product of the

rotation group $S O(2)$ and the plane translation group, $T$. Its unitary representation in the 
Hilbert space $L^{2}\left(\mathbb{R}^{2}\right)$ of square integrable complex functions on the plane accounts for the free particle case $(B=0)$ while its non-trivial PUR's account for the non-zero magnetic field case. Since we will use this approach to consider electron on a cylinder, we will first discuss briefly the usual Landau problem from the symmetry point of view. It turns out that for the classification of the PURs of $E_{2}$, it is sufficient to concentrate on the subgroup of translations, $T$.

\section{A. Preliminaries}

Let us briefly recall some basic definitions and facts about PURs and central extensions of a general Abelian group, $G$. In the following, the group multiplication in $G$ is denoted additively.

A PUR of $G$ is a UR up to a phase i.e. if $U$ is a PUR of $G$, then $U(g) U(h)=\gamma(g, h) U(g+$ h) $\forall g, h \in G$, where $\gamma(g, h)$ is a 2-cocycle, namely, a complex valued function with absolute value 1 , satisfying certain conditions. If $\gamma$ is of the form $\gamma(g, h)=\delta(g) \delta(h) \delta(g+h)^{-1}$ with $\delta$ mapping the group elements to complex numbers of modulus 1 , then it is a coboundary and the corresponding PUR is a UR of $G$.

PURs of $G$ are more conveniently described in terms of the so-called group commutator of $U(g)$ and $U(h)$,

$$
c(g, h):=U(g) U(h) U(g)^{-1} U(h)^{-1}=\gamma(g, h) \gamma(h, g)^{-1}, \forall g, h \in G .
$$

We shall call $c$ the commutator function on $G \times G$ corresponding to the PUR $U$. The definition of a PUR implies that $c$ satisfies:

$$
c(g+h, k)=c(g, k) c(h, k), c(g, h+k)=c(g, h) c(g, k), c(g, h) c(h, g)=1 .
$$

The functions $c$ on $G \times G$ satisfying (2) characterize fully equivalence classes of PURs of

$G$ : the identity function $c=1$ corresponds to the class of trivial PURs, namely the URs of $G$ and the commutator function is unchanged when a 2-cocycle is modified by a coboundary. When $G$ is a Lie group and the PUR is a continuous one (the only case of relevance here) one can write

$$
c(g, h)=e^{i \alpha(g, h)}
$$

where $\alpha$ is a real valued, continuous, bilinear, antisymmetric function of its arguments. To find all PURs of $G$ one needs to obtain all such $\alpha$ 's and find continuous maps $U$ from $G$ 
into unitary operators on some Hilbert space $\mathcal{H}$ satisfying (11) for each $\alpha$. If there are no non-zero $(\bmod 2 \pi) \alpha$ 's, then $G$ has no non-trivial PURs.

As a general example and one which we will use here, take $G$ to be the direct product of an Abelian group $A$ and its group of irreducible characters (its Pontryagin dual), $A^{*}$ i.e. $G=A \times A^{*}$. Given any two characters $\chi, \phi$ of $A$, define

$$
c((g, \chi),(h, \phi)):=\chi^{-1}(h) \phi(g) \quad \forall(g, \chi),(h, \phi) \in A \times A^{*} .
$$

It is easy to see that this function defines a commutator function for $G$. Thus every pair of characters of $A$ defines a class of PURs of $A \times A^{*}$.

Fix a $c=e^{i \alpha}$ corresponding to a PUR of a general Abelian group, $G$ and consider the set of ordered pairs $(g, s)$ of elements of $G$ and $U(1)$. Define the composition law,

$$
(g, s) \cdot(h, t):=\left(g+h, e^{i \beta(g, h)} s t\right), \text { such that } \alpha(g, h)=\beta(g, h)-\beta(h, g) .
$$

From Eq.(11), we can write a cocycle corresponding to $c$ as $\gamma(g, h)=e^{i \beta(g, h)}$. But $\beta$ is not uniquely defined by (5). In many (but not all [4]) cases, we can choose $\beta$ itself to be antisymmetric: $\beta=\frac{1}{2} \alpha$, which is a canonical choice. In the physical context, this will be seen to correspond to working in the symmetric gauge. We will see in section IIE that this canonical choice is impossible for the periodic cylinder forcing us to work in a Landau-like gauge.

It follows that this composition makes the set of ordered pairs into a (non-Abelian) group which is denoted as $G \times{ }_{c} U(1)$ or $\tilde{G}_{c}$. This group has $U(1)$ as a central subgroup such that $\tilde{G}_{c} / U(1) \sim G$ : it is a central extension of $G$ by $U(1)$. If and only if, $c=1$ identically, the corresponding central extension is trivial, i.e. $\sim G \times U(1)$ and $G$ is a subgroup of $\tilde{G}_{c}$. It is easy to verify that the group commutator in $\tilde{G}_{c}$ is given by,

$$
(g, s) \cdot(h, t) \cdot(g, s)^{-1} \cdot(h, t)^{-1}=(0, c(g, h))
$$

independent of $s, t$, and so determines a commutator function on $G$.

Now consider a unitary representation $\tilde{U}$ of $\tilde{G}_{c}$ such that $\tilde{U}(0, s)=s$. Then it follows that

$$
\tilde{U}(g, s) \tilde{U}(h, t) \tilde{U}(g, s)^{-1} \tilde{U}(h, t)^{-1}=c(g, h) .
$$

In particular, $U(g):=\tilde{U}(g, 1)$ is a PUR of $G$ corresponding to the commutator function c. The important result is that every PUR of $G$ arises this way from a UR of one of its 
central extensions. For further details we refer the reader to [4]. One physically significant consequence of these facts is that the group that acts linearly (unitarily) on the Hilbert space of a system having symmetry group $G$ is, in general, not $G$ itself but some central extension $\tilde{G}$ of $G$ by $U(1)$.

\section{B. The Landau electron}

We expect the symmetry group of a charged particle moving on the plane with a uniform magnetic field $B$, perpendicular to it to be the Euclidean group, $E_{2}$. To apply the general procedure outlined above to this case, we note that central extensions of $E_{2}$ are completely determined by those of its translation subgroup $T[5] . T$ is the group of two dimensional vectors, $\vec{x}, \vec{y}, \cdots$, under addition. It is also the configuration space of the particle. A general real, bilinear, antisymmetric function of $\vec{x}, \vec{y}$ is clearly of the form:

$$
\alpha_{\lambda}(\vec{x}, \vec{y})=\lambda \vec{x} \wedge \vec{y}:=\lambda\left(x_{1} y_{2}-x_{2} y_{1}\right)
$$

where $\lambda$ is any real number. It is easy to see that when the group commutator defined by the extension $\tilde{T}_{\lambda}$ is specialized to group elements close to the identity, one gets the Lie algebra bracket among the generators $\left(P_{1}, P_{2}\right.$ forming the basis of the Lie algebra) of $\tilde{T}_{\lambda}$ as:

$$
\left[P_{1}, P_{2}\right]=i \lambda
$$

Note that $\tilde{T}_{\lambda}$ has one extra generator (corresponding to the $U(1)$ ) which commutes with the remaining generators and can be taken to be the identity operator in any irreducible UR, $\tilde{U}$ of interest, since $\tilde{U}(0, s)=s$. This is suppressed in the equations. A non-zero $\lambda$ labels equivalence classes of non-trivial PURs of the translation subgroup while $\lambda=0$ corresponds to the URs. As stated earlier, every central extension $\tilde{T}_{\lambda}$ extends to a central extension of the Euclidean group and all central extensions of $E_{2}$ arise this way. $\tilde{T}_{\lambda \neq 0}$ is the familiar Heisenberg group as is apparent from the commutator between $P_{i}$. As is well known, this has a unique (up to unitary equivalence), continuous, irreducible, infinite dimensional, unitary representation. The most familiar concrete form of this UR, from the quantum mechanics of a particle in one dimension, is on $L^{2}(\mathbb{R})$.

Since our configuration space is $\mathbb{R}^{2}$, we are interested in the UR of $\tilde{T}_{\lambda}$ on $L^{2}\left(\mathbb{R}^{2}\right)$ and this UR, called the wave function UR, is not irreducible. Again the general theory [6] implies 
that this particular representation is precisely characterized as the unique, irreducible UR of $\tilde{T}_{\lambda} \times \tilde{T}_{-\lambda}$. Equivalently, $L^{2}\left(\mathbb{R}^{2}\right) \sim \mathcal{V}_{\lambda} \otimes \mathcal{V}_{-\lambda}$ where $\mathcal{V}_{ \pm \lambda}$ are the representation spaces of the irreducible URs of $\tilde{T}_{ \pm \lambda}$. Explicitly, the wave function representation is obtained as,

$$
\left(W_{\lambda}(\vec{x}, \vec{y}) \psi\right)(\vec{w}):=e^{i \frac{\lambda}{2}(\vec{x} \wedge \vec{w}-\vec{y} \wedge \vec{w})} \psi(\vec{w}+\vec{x}+\vec{y})
$$

Here $(\vec{x}, 1) \in \mathbb{R}^{2} \times_{\lambda} U(1),(\vec{y}, 1) \in \mathbb{R}^{2} \times_{-\lambda} U(1)$ and $\vec{w} \in \mathbb{R}^{2}$ and verification is a matter of simple algebra.

In the present subsection, we have adopted the canonical choice of the cocycle $\beta=\frac{1}{2} \alpha$. For the real Heisenberg group, this is always possible. When the identification with the plane Landau problem is made, a choice of the cocycle is the same as fixing the gauge. The above canonical choice corresponds to the symmetric gauge. Thus $\psi$ in (10) is a symmetric gauge wavefunction.

This completes the description of the kinematics of the projectively realized translation symmetry of a particle in $\mathbb{R}^{2}$, namely, the specification of the state space $\mathcal{H}_{\lambda}=L^{2}\left(\mathbb{R}^{2}\right)$ and the action of the symmetry group on it via an irreducible UR of $\tilde{T}_{\lambda} \times \tilde{T}_{-\lambda}$. We emphasize that the symmetry group gives rise to a central extension $\tilde{T}_{\lambda}$ acting unitarily on $\mathcal{V}_{\lambda}$ while the second factor $\tilde{T}_{-\lambda}$ keeps track of the multiplicity of the irreducible UR of $\tilde{T}_{\lambda}$ in the state space $\mathcal{H}_{\lambda}$.

To specify dynamics, we need to pick an operator $H_{\lambda}$ on $\mathcal{H}_{\lambda}$, the Hamiltonian, which is invariant under the action of $\tilde{T}_{\lambda}$ (but not under the action of $\tilde{T}_{-\lambda}$ ). From the commutation relations between the momenta $P_{i}$, it is clear that no polynomial in these can be so invariant and hence the Hamiltonian cannot be a polynomial in $P_{i}$; the Hamiltonian must be an operator of the form $1 \otimes \mathcal{O}$. We have however the position operators $X_{1}, X_{2}$ on the Hilbert space. Consider the following combinations (we have set $\hbar=1$ ):

$$
K_{1}:=P_{1}-\lambda X_{2}, \quad K_{2}:=P_{2}+\lambda X_{1}
$$

These satisfy,

$$
\left[K_{1}, K_{2}\right]=-i \lambda, \quad\left[K_{i}, X_{j}\right]=-i \delta_{i j}, \quad\left[K_{i}, P_{j}\right]=0 .
$$

So $K_{i}$ form a basis for the Lie algebra of $\tilde{T}_{-\lambda}$ and hence map $\mathcal{V}_{-\lambda}$ to itself. Since the actions of $P_{i}$ and $K_{i}$ commute in $\mathcal{H}_{\lambda}$, a polynomial in $K_{i}$ is a candidate for a Hamiltonian. A simple choice, consistent with the rotational symmetry is:

$$
H_{\lambda}:=\frac{1}{2 m}\left(K_{1}^{2}+K_{2}^{2}\right) \text {. }
$$


This immediately leads to,

$$
\begin{aligned}
V_{i}:=\frac{d X_{i}}{d t} & =i\left[H_{\lambda}, X_{i}\right]=K_{i} / m \\
m \frac{d^{2} X_{i}}{d t^{2}} & =i\left[H_{\lambda}, K_{i}\right]=-\lambda \epsilon_{i j} K_{j} .
\end{aligned}
$$

Thus $K_{i}$, which is not the momentum, is actually proportional to the velocity. Identifying $\lambda=e B$, we see that the Hamiltonian is exactly that for a charged particle in a plane with a uniform magnetic field perpendicular to the plane. The commutation relations between the velocity operators, $K_{i}$, lead to the usual spectrum of the Landau problem with $\operatorname{dim}\left(\mathcal{V}_{\lambda}\right)=\infty$ as the degeneracy. The usual degeneracy is thus manifestly a consequence of the translation symmetry which is however realized projectively.

We also note here the physical meaning of the commutator function:

$$
\alpha_{\lambda}(\vec{x}, \vec{y})=e B \vec{x} \wedge \vec{y}=: e \Phi(\vec{x}, \vec{y})=2 \pi \Phi(\vec{x}, \vec{y}) / \Phi_{0}
$$

where $\Phi(\vec{x}, \vec{y})$ is the flux of $B$ through the parallelogram of sides $\vec{x}, \vec{y}$ and $\Phi_{0}=\frac{2 \pi}{e}$ is the value of the flux quantum.

This demonstrates that the translation symmetric quantum mechanics of a particle in the plane covers not only free motion $(\lambda=0)$ but also the Landau electron $(\lambda=e B)$. The belief that the fundamental physical quantity is the field itself - which is translationally invariant rather than the vector potential - which is not invariant - is thereby vindicated. Gauges and gauge transformations turn out to be artifacts of the many equivalent ways of choosing the 2-cocycle and of constructing the unique irreducible URs of $\tilde{T}_{\lambda}[\underline{5}]$ and can be very naturally accommodated.

Our treatment of the particle on a cylinder from both the representation theoretic and the 'geometric' viewpoint will highlight the care needed in dealing with a topologically nontrivial configuration space. Once that is done, both approaches will lead to exactly the same conclusions.

\section{Magnetic field on a cylinder}

We now attempt to carry over the representation-theoretic method to the motion of a charged particle on the surface of an infinite cylinder $S^{1} \times \mathbb{R}$ in the presence of a uniform, radial magnetic field, $B$. The physical realization of such a magnetic field is of no concern 
to us here. The group of symmetry transformations, called the cylinder group, is $C:=$ $S O(2) \times \mathbb{R}$. Topologically, $S O(2)$ is of course $S^{1}$. For clarity we denote the circle as a manifold by $S^{1}$, as a transformation group by $S O(2)$ and as the multiplicative group of phases by $U(1)$, as we have already done. The magnetic field of course does not break the symmetry under $C$.

If the corresponding quantum theory can be fully formulated in terms of $B$, without invoking a vector potential, then we expect it to be describable, exactly as before, by continuous PURs of the cylinder group. If not, then the symmetry under $C$ is necessarily broken.

The striking fact is that the cylinder group has no continuous, nontrivial, PURs at all! This follows directly from the general form of the commutator function, $c(g, h)$, given in (3). Denoting a group element in $C$ as a pair $(\theta, \eta)$ with $\theta \in S O(2)$ and $\eta \in \mathbb{R}$, the commutator functions of $C$ must be of the form $e^{i \lambda\left(\theta \eta^{\prime}-\theta^{\prime} \eta\right)}$. The only way this can be invariant under the $2 \pi$ periodicity of the angles $\theta, \theta^{\prime}$ for all $\eta, \eta^{\prime}$ is if $\lambda$ is zero.

It is to be stressed that if we were to replace the cylinder group $C$ by its universal covering group $\bar{C} \sim \mathbb{R}^{2}$, or look only at its Lie algebra (also $\mathbb{R}^{2}$ ), this negative result no longer holds. Effectively, such a replacement amounts to replacing the configuration space $S^{1} \times \mathbb{R}$ by $\mathbb{R}^{2}$ and this is unjustified. Indeed, it is the nontrivial topology of the cylinder group which in turn is dictated by the topology of the configuration space that is responsible for the non-existence of nontrivial PURs of $C$. The true symmetry group of the cylinder is $C$ and not $\bar{C}$. The question of when a symmetry group can be replaced by its universal cover for quantum mechanical purposes is discussed at length in [3].

Physically this means that there is no way of constructing quantum mechanics of a charged particle on a cylinder in the presence of a uniform radial magnetic field if the state space is to carry a PUR of the translation group $C$ of the cylinder. This is a rather strong conclusion as it does not depend on any a priori assumptions about the details of the function space (with suitable boundary conditions) to serve as the state space, leave alone the specific choice of the Hamiltonian as an operator on that space. It does not negate the value of a formulation of the theory depending essentially on the vector potential, but we should not then expect to fully implement the translation invariance. In the next section a treatment based on vector potential will be presented with essentially the same conclusion, that the full translation symmetry is not possible. 
We have already noted that infinitesimal translations, encoded in the Lie algebra of $C$, and hence not distinguishing between the cylinder and the plane, do have non-trivial PURs. This has a useful consequence. We can "integrate" a PUR of $C$ along any curve which does not fully wind around the cylinder. More precisely, consider a contractible region $D$ of $S^{1} \times \mathbb{R}$. Suppose $D$ contains the 'rectangle' with vertices at $(0,0),(\theta, 0),(\theta, \eta)$ and $(0, \eta)$. Then from the Lie algebra central extension

$$
\left[P_{\theta}, P_{\eta}\right]=i \lambda
$$

we have the group commutator,

$$
e^{i \theta P_{\theta}} e^{i \eta P_{\eta}} e^{-i \theta P_{\theta}} e^{-i \eta P_{\eta}}=e^{i \lambda \theta \eta}
$$

If we apply a uniform radial magnetic field to a particle moving in $D$, then its momentum will satisfy these equations with the identification $\lambda=e B$, exactly as in the case of the plane eq.(16). Hence, locally within $D$, we have the old relationship

$$
e^{i \theta P_{\theta}} e^{i \eta P_{\eta}} e^{-i \theta P_{\theta}} e^{-i \eta P_{\eta}}=e^{i e B \theta \eta}=: e^{2 \pi i \frac{\Phi(\theta, \eta)}{\Phi_{0}}}
$$

where $\Phi(\theta, \eta)$ is the flux through the rectangle and $\Phi_{0}$ is the flux quantum. This will break down as soon as $D$ (and the rectangle in it) becomes non-contractible.

\section{The Periodic cylinder}

While the cylinder group has no nontrivial PURs, it has a subgroup which does. One can apply our methods to specify the state space and the dynamics of the Landau electron having the reduced symmetry of the subgroup.

The key fact is that the subgroup $C_{\mathbb{Z}}:=S O(2) \times \mathbb{Z}$ of $C$, has the structure $A \times A^{*}$. This is because the group of characters of $S O(2)$ is $\mathbb{Z}$ and vice versa, i.e. $S O(2)^{*}=\mathbb{Z}$ and $\mathbb{Z}^{*}=S O(2)$. From the general theory summarized in $\llbracket \mathrm{A}, C_{\mathbb{Z}}$ has a Heisenberg central extension given by the commutator function,

$$
c_{\nu}\left((\phi, m),\left(\phi^{\prime}, m^{\prime}\right)\right):=e^{i \nu\left(m^{\prime} \phi-m \phi^{\prime}\right)},
$$

where $\nu$ is an integer since $\phi$ is an angle.

Physically one may imagine reducing $C$ to $C_{\mathbb{Z}}$ by applying a periodic potential $V$, along the axial direction: $V$ is independent of $\phi$ and $V(\eta+\ell)=V(\eta)$ for a fixed $\ell$ which we take 
to be 1 . Such a potential will have to be added to the Hamiltonian, but it can be taken to be arbitrarily small. Its only purpose here is to reduce the symmetry group.

In looking for the quantum mechanical realization of the PURs of $C_{\mathbb{Z}}$ through a magnetic field $B$, it is to be expected that there may be restrictions on the possible values of $B$, arising from the quantization of the central charge $\nu$. This in fact is the case. From eq.(20), we have the condition

$$
c_{\nu}((2 \pi, 0),(0,1))=1
$$

for the commutator of a translation through a period along the axis and by $2 \pi$ around the axis implying that $\nu$ is an integer. But from the eq.(19), in the limit $\phi \rightarrow 2 \pi$ and $\eta=1$, we also have

$$
c_{\nu}((2 \pi, 0),(0,1))=e^{i 2 \pi \frac{\Phi}{\Phi_{0}}}
$$

where $\Phi$ is the flux through a unit slice of the cylinder. We conclude:

The periodic cylinder group $C_{\mathbb{Z}}=S O(2) \times \mathbb{Z}$ has a family of PURs labelled by integers, describing at least at the kinematical level, the quantum mechanics of a charged particle in a uniform radial magnetic field $B$ if and only if $B$ is such that the flux per slice is an integral multiple of the flux quantum.

In this form the conclusion is dimension-free: it is independent of the radius of the cylinder and the period of the periodic potential. The corresponding central extensions $C_{\mathbb{Z}} \times_{\nu} U(1)$ are classified by the quantized values of the flux/slice. The only 'magnetic quantity' that enters in the description is the flux/slice and not $B$ itself.

\section{E. Representations, wave functions, dynamics}

It is convenient to borrow from the mathematics literature the term "Heisenberg group" to designate a nontrivial central extension by $U(1)$ of an Abelian group $G$ having the structure $A \times A^{*}[6]$. The distinguishing feature of a Heisenberg group is the Stone-von Neumann property: all its continuous irreducible unitary representations are unitarily equivalent. The essentially unique irreducible UR can be concretely realized on $L^{2}$ functions on certain quotient groups of $G$ (all of which are equivalent), in particular, on $L^{2}(A)$ and on $L^{2}\left(A^{*}\right)$. The "original" Heisenberg group $\mathbb{R}^{2} \times_{\lambda} U(1), \lambda \in \mathbb{R}$ provides a familiar illustration of all these properties. 
The centrally extended periodic cylinder group $\tilde{C}_{\mathbb{Z} \nu}:=(S O(2) \times \mathbb{Z}){ }_{\nu} U(1)$ is a Heisenberg group and has equivalent irreducible URs on $L^{2}\left(S^{1}\right)$ and $L^{2}(\mathbb{Z})$.

On $L^{2}\left(S^{1}\right)$, the action of $\tilde{C}_{\mathbb{Z} \nu}$ is given by

$$
\begin{aligned}
\left(U_{S^{1}}(\theta, 0) f\right)(\phi) & :=f(\theta+\phi) \\
\left(U_{S^{1}}(0, m) f\right)(\phi) & :=c_{\nu}((\phi, 0),(0, m)) f(\phi)=e^{i \nu m \phi} f(\phi) .
\end{aligned}
$$

Likewise, on $L^{2}(\mathbb{Z})$, the action of $\tilde{C}_{\mathbb{Z} \nu}$ is given by

$$
\begin{aligned}
\left(U_{\mathbb{Z}}(\theta, 0) f\right)(n) & :=c_{\nu}((0, n),(\theta, 0)) f(n)=e^{-i \nu n \theta}, \\
\left(U_{\mathbb{Z}}(0, m) f\right)(n) & :=f(n+m) .
\end{aligned}
$$

For the wave function representation, we look for representations of the symmetry group $\tilde{C}_{\mathbb{Z} \nu}$ on square integrable functions, or more generally on $L^{2}$ sections of a line bundle, on the configuration space $S^{1} \times \mathbb{R}$. These are characterized by a quasi-periodicity parameter $q$ defined as $\psi(\theta+2 \pi, y)=e^{2 \pi i q} \psi(\theta, y)$ and the Hilbert space is denoted by $\mathcal{H}_{\nu}:=L_{q}^{2}\left(S^{1} \times \mathbb{R}\right)$. The UR $W_{\nu}$ of $\tilde{C}_{\mathbb{Z} \nu}$ is explicitly defined for a fixed $q$ as:

$$
\left(W_{\nu}(\phi, m) \psi\right)(\theta, y):=e^{i(\nu m \theta-q \phi)} \psi(\theta+\phi, y+m)
$$

The phase factor in (25) corresponds to a choice of cocycle which is not the canonical one. The reason is that the square root of the commutator function is not well defined on $C$. It is easily checked that this choice is compatible with the commutator function in (201). One can also see that $W_{\nu}(2 \pi, 0)=\mathrm{Id}$ on the state space $\mathcal{H}_{\nu}$. This representation of $\tilde{C}_{\mathbb{Z} \nu}$ cannot be irreducible.

Remarkably, this space $\mathcal{H}_{\nu}$ also carries a UR, $V_{-\nu}$, of the real Heisenberg group corresponding to central charge $-\nu$. This is defined explicitly as:

$$
\left(V_{-\nu}(\xi, \eta) \psi\right)(\theta, y):=e^{i \nu \xi y} \psi(\theta+\xi, y+\eta)
$$

Furthermore, the actions of the two groups commute. The phase factor in (26) corresponds to a choice of cocycle which is also not the canonical one. It is for this choice that the two group actions commute. Once again, the commutator function given in (요 is unchanged. These facts are easily checked.

It follows from this that state space has the decomposition:

$$
\mathcal{H}_{\nu}=\mathcal{W}_{\nu} \otimes \mathcal{V}_{-\nu}
$$


where $\mathcal{W}_{\nu}$ and $\mathcal{V}_{-\nu}$ are representation spaces for $W_{\nu}$ and $V_{-\nu}$ respectively.

For every fixed $q$, one also has the decomposition, $L_{q}^{2}\left(S^{1} \times \mathbb{R}\right)=L_{q}^{2}\left(S^{1}\right) \otimes L^{2}(\mathbb{R})$. This fact together with eq.(27) implies that $\mathcal{H}_{\nu}$ is an irreducible UR of $\tilde{C}_{\mathbb{Z} \nu} \times \tilde{\mathbb{R}}_{-\nu}^{2}$, since each factor in the tensor product is irreducible under the corresponding subgroup.

We emphasize that the first factor in the tensor product decomposition of the state space represents the symmetry group while the second factor represents the multiplicity of the representation. The second factor is where the dynamics will be defined.

As before, the only operators invariant under the symmetry group $\tilde{C}_{\mathbb{Z} \nu}$ are of the form $1 \otimes \mathcal{O}$ and the correct invariant Hamiltonian is:

$$
H_{\nu}=1 \otimes \frac{\left(K_{1}^{2}+K_{2}^{2}\right)}{2 m}
$$

with $\left[K_{1}, K_{2}\right]=-i \nu=-i e B_{\nu}$ and $B_{\nu}$ such that the flux $2 \pi B_{\nu}=\nu \Phi_{0}$. Again, as before, one verifies that $K_{i}$ are indeed the velocity operators. Strictly speaking we also have to add a periodic potential needed to reduce the symmetry group. The strength of this potential can be taken to be arbitrarily small so that it defines an "empty lattice" along the axis of the cylinder.

In the empty lattice limit, the spectrum of the Hamiltonian is exactly as in the plane. This is not surprising since velocities are tangent to the cylinder and so are insensitive to whether one is working on the plane or the cylinder. The one difference is that not only is the energy quantized as a function of the magnetic field but the flux itself can only take allowed quantized values for which PURs of $C_{\mathbb{Z}}$ exist. Every energy eigenvalue has multiplicity 1 in $\mathcal{V}_{-\nu}$ while $\mathcal{W}_{\nu}$ accounts for the infinite degeneracy of the energy eigenvalues in the full state space, exactly as in the plane problem.

\section{THE HAMILTONIAN APPROACH}

In the previous section we saw the construction of the quantum mechanics (state space and dynamics) of a charged particle in a uniform magnetic field first on a plane and then on a cylinder. The distinctive feature of that approach was that a symmetry group was chosen a priori, as a group of transformations of the configuration space of the system under which the specification of the system was invariant, namely the Euclidean group in the case of the plane and the cylinder group, $S O(2) \times \mathbb{R}$, in the case of the cylinder. Although the 
system was 'obviously' invariant under these groups, surprisingly at the quantum level the symmetry was reduced to $S O(2) \times \mathbb{Z}$ in the case of the cylinder while in the case of the plane, there was no such reduction of the symmetry. This followed from the non-existence of non-trivial, projective unitary representations of the cylinder group. In this analysis, the Hamiltonian played no explicit role and was in fact derived by demanding invariance under the symmetry action. There was no explicit introduction of a gauge potential, and the approach was manifestly gauge invariant.

In this section we will arrive at the same conclusions using the more familiar approach in which the Hamiltonian is chosen to begin with and the corresponding admissible symmetries are deduced as a consequence. This will also serve to clarify the surprising nature of the result. In brief, it will turn out that, in a certain sense, the full cylinder symmetry was never there to begin with! We will concentrate only on the cylinder case with reference to the plane only for comparison. Throughout this section, physical units are used in order to highlight the points at which quantum considerations come into play.

\section{A. Key points and the basic set up}

It is worth keeping in mind the following three points to appreciate the steps of the arguments leading to the result. These points are elaborated further subsequently.

(1) Since in the present approach, we begin with an a priori chosen classical Hamiltonian corresponding to the interaction of a charged particle with an external magnetic field, we will have to introduce a gauge potential to be able to write the Hamiltonian in a local form. The same of course is also true in a formulation based on an action principle. One then has the freedom to choose any of the gauge potentials which give the same magnetic field. While stipulating the class of transformations of the cylinder which qualify to be symmetries within the formulation, one has to pay attention to this freedom.

(2) The topology of the cylinder implies that there are several gauge potentials, giving the same magnetic field, which however do not differ by the gradient of functions on the cylinder. These are classified further by the notion of 'holonomy equivalence'. It will turn out that there are infinitely many holonomy equivalence classes on the cylinder while there is only one such class on the plane. A complete gauge invariant specification of the system is made by giving a single holonomy equivalence class. 
(3) At the quantum mechanical level, in the context of a Schrödinger like representation, the wave functions (sections of line bundles) have to be chosen to be quasi-periodic. As usual, functions of the coordinates will act by multiplication. To preserve the quasi-periodicity of the wave functions, such multiplicative operators must be single valued. In particular the gauge potentials must be single valued.

The cylinder is taken to be coordinatized in the usual manner by the angular coordinate $\theta$ and the coordinate $y$ along the axis. The corresponding conjugate momenta are $p_{\theta}, p_{y}$ and the radius of the cylinder is $R$. The classical Hamiltonian is given by (we have chosen the units so that the speed of light in vacuum is 1 ),

$$
H\left(\theta, y, p_{\theta}, p_{y} ; A(\theta, y)\right):=\frac{\left(p_{y}-e A_{y}(\theta, y)\right)^{2}}{2 m}+\frac{\left(p_{\theta}-e A_{\theta}(\theta, y)\right)^{2}}{2 m} R^{-2}
$$

Quantum mechanically, this is to be represented by a self adjoint operator on a suitable Hilbert space, with the conjugate momenta acting as derivative operators and coordinates acting as multiplication operators. Explicitly, $p_{y}:=-i \hbar \partial_{y}, p_{\theta}:=-i \hbar \partial_{\theta}$. To ensure that the momenta, in particular $p_{\theta}$, are self adjoint, their domain must consist of wave functions $\psi(\theta, y)$ which are in general quasi-periodic: $\psi(\theta+2 \pi, y)=e^{2 \pi i q} \psi(\theta, y)$, where $q$ is a real parameter in the interval $[0,1)$, specifying the quasi-periodicity. For the Hamiltonian to be a well defined operator on such quasi-periodic functions then, the gauge potential must be single valued as already remarked.

\section{B. Classification of gauge potentials}

Let us now turn to gauge transformations. Recall that the usual gauge transformations of the gauge potentials and the phase transformations of the quantum wave function are correlated in the following manner. Using the notation, $g:=e^{i \frac{e}{\hbar} \Lambda}$,

$$
\begin{aligned}
& \psi^{\prime}:=g \psi, \quad A_{j}^{\prime}:=g A_{j} g^{-1}+i \frac{\hbar}{e} g \partial_{j} g^{-1} \\
&\left\{-i \hbar \partial_{j}-e A_{j}^{\prime}\right\} \psi^{\prime}=g\left\{-i \hbar \partial_{j}-e A_{j}\right\} \psi
\end{aligned}
$$

The phase transformation $g$, also has to be single valued to preserve the quasi-periodicity of the wave functions. This also preserves the single valued nature of the gauge potential. Notice that if $\Lambda$ is single valued, so is $g$ but the converse is not true. For example $g_{m}(\theta, y):=$ $e^{i m \theta}, m \in \mathbb{Z}$, is single valued although $\Lambda \sim \theta$ is not. Incidentally, these are the only phase transformations which are single valued without the corresponding exponent $i \frac{e}{\hbar} \Lambda$ being so. 
It is convenient to use the differential form notation: $A:=A_{y} d y+A_{\theta} d \theta, d:=d y \partial_{y}+$ $d \theta \partial_{\theta}, d A=\left(\partial_{\theta} A_{y}-\partial_{y} A_{\theta}\right) d \theta \wedge d y$ etc. In the present context of a uniform radial magnetic field of magnitude $B$, we have $d A=B R d \theta \wedge d y$.

In terms of this notation, two gauge potential 1-forms, $A^{\prime}, A$, are defined to be gauge equivalent if they are related by a gauge transformation,

$$
A^{\prime}=A^{g}:=g A g^{-1}+i \frac{\hbar}{e} g d g^{-1}=A+i \frac{\hbar}{e} g d g^{-1}
$$

for some smooth, single valued function $g$ on the cylinder. It is immediate that the corresponding magnetic field 2-forms, $d A^{\prime}, d A$ are equal for all gauge equivalent potentials, expressing the gauge invariance of the magnetic field. Note that the converse need not be true i.e. we may have two gauge potentials giving the same magnetic field which are nonetheless not gauge equivalent.

To see this, observe that the difference of any two gauge potentials giving the same magnetic field must be a closed 1-form. On the plane, it being contractible, every closed 1-form is also exact i.e. it is the exterior derivative (or gradient) of some function $\Lambda$ on the cylinder. It is obvious then that the two potentials are related by a gauge transformation. There is thus exactly one gauge equivalence class of the potential and it is characterized by the magnetic field. The situation on the cylinder is different. Every closed 1-form is not exact. Since the cylinder is simple enough we can write down the most general form of a gauge potential:

$$
A^{\prime}=A+\zeta d \theta+d \Lambda \quad \Longleftrightarrow \quad A_{\theta}^{\prime}=A_{\theta}+\partial_{\theta} \Lambda+\zeta, A_{y}^{\prime}=A_{y}+\partial_{y} \Lambda
$$

Here $\zeta$ is a real constant and $\Lambda$ is of course a function on the cylinder which by definition is single valued. A convenient parameterization of all the gauge potentials giving the same magnetic field is then obtained by choosing $A_{y}=0, A_{\theta}=-B y$ so that,

$$
A^{\zeta}=(\zeta-B R y) d \theta+d \Lambda, \quad d A^{\zeta}=B(R d \theta \wedge d y) .
$$

Note that $A^{\zeta}$ and $A^{\zeta^{\prime} \neq \zeta}$ are not gauge equivalent on the cylinder since $\theta$ is not a single valued function (or a 0-form) on the cylinder. Incidentally single valuedness of gauge transformation also implies that unlike the planar case, a 'symmetric' gauge for the gauge potential $\left(A_{y}=\frac{B}{2} R \theta, A_{\theta}=-\frac{B}{2} R y\right)$ is not possible. At this stage we have a 1-parameter family of gauge equivalence classes of the potentials, all giving the same magnetic field. Clearly, 
unlike on the plane, the magnetic field alone does not constitute a complete characterization of a gauge equivalence class.

However, it is well known that there is another way to classify gauge potentials using their holonomies (also known as exponentials of non-integrable phase factors [7]) along all closed curves (or loops for short). Since on a cylinder we do have non-contractible loops, those which wind around the cylinder, we do have a 'larger' class of holonomies compared to the planar case.

The holonomy of a gauge potential (in the Abelian case) along a loop $\tau$ is defined to be:

$$
h_{\tau}(A):=\exp \left(i \frac{e}{\hbar} \oint_{\tau} A\right)
$$

One can now define a holonomy equivalence relation on the set of gauge potentials. Two gauge potentials are said to be holonomically equivalent if their holonomies along all loops are equal. In the present context we will see explicitly that holonomy equivalence implies gauge equivalence and conversely.

Using the parameterization of the gauge potential (133), it is easy to see that

$$
\begin{aligned}
h_{\tau}^{\zeta} & =\exp \left\{i \frac{e}{\hbar} \oint \zeta d \theta+i \frac{e}{\hbar}(-B R) \oint y d \theta+i \frac{e}{\hbar} \oint d \Lambda\right\} \\
& =\exp \left\{i \frac{e}{\hbar} \zeta 2 \pi w_{\tau}\right\} \exp \left\{-i \frac{e}{\hbar} \Phi_{\tau}\right\}
\end{aligned}
$$

where $w_{\tau}$ is the number of times the loop $\tau$ winds around the cylinder and $w_{\tau}=0$ corresponds to a contractible loop. For a contractible loop, the second factor contains the flux of the magnetic field through the loop. The first factor contains the dependence on the parameter $\zeta$ while the second one is common to all gauge potentials of eq.(33) and is manifestly gauge invariant.

It follows immediately that

$$
h_{\tau}^{\zeta^{\prime}}=h_{\tau}^{\zeta} \exp \left\{i \frac{e}{\hbar}\left(\zeta^{\prime}-\zeta\right) 2 \pi w_{\tau}\right\}
$$

Clearly, for all contractible loops the holonomies of all $A^{\zeta}$ are equal. For non-contractible loops though the holonomies are equal if and only if

$$
\zeta^{\prime}=\zeta+m\left(\frac{\hbar}{e}\right), m \in \mathbb{Z} .
$$

Now, if two gauge potentials are gauge equivalent, their difference is proportional to $g d g^{-1}$ which is either an exact 1-form or is proportional to $d \theta$. In either case, their holonomies 
along all loops are equal. Thus gauge equivalence implies holonomy equivalence. To prove the converse, we use the equation (37) and the parameterization in (33). It then follows that we can first choose a $g_{m}=e^{i m \theta}$ to make the two $\zeta$ 's equal and then choose a $\Lambda$ to make the two potentials equal. Thus we see that holonomy equivalence also implies gauge equivalence. From now on we will refer to them simply as gauge equivalence classes and denote them as $\left[A^{\zeta}\right]$ :

$$
\left[A^{\zeta}\right]:=\left\{A^{\zeta^{\prime}} / A^{\zeta^{\prime}}=\left(\zeta^{\prime}-B R y\right) d \theta+d \Lambda, \zeta^{\prime}=\zeta+m \frac{\hbar}{e}, m \in \mathbb{Z}\right\}
$$

The gauge equivalence classes of gauge potentials are thus labeled by a real number in the interval $\left[0, \frac{\hbar}{e}\right)$; shifting $\zeta$ by an integer multiple of $\frac{\hbar}{e}$ keeps the gauge potential within the same gauge equivalence class. The holonomies are functions of the equivalence classes.

\section{Definition of symmetry transformations}

Having specified the quasi-periodicity properties of the wave functions, the action of gauge transformations on them and classified the gauge potentials, we now turn to the specification of the symmetry transformations.

The classical equations of motion are of course manifestly gauge invariant. However, due to the explicit presence of the gauge potential, the Hamiltonian is manifestly gauge non-invariant. In the usual context of ordinary potentials, symmetry transformations are required to leave the Hamiltonian invariant. In the present context, we must allow the Hamiltonian to change while formulating the definition of a symmetry transformation. The allowed change in the Hamiltonian is not arbitrary but must be such that it can be compensated by a gauge transformation of the gauge potential.

Thus we define symmetry transformations as those transformations of the configuration space which induce a gauge transformation on the gauge potential.

This is physically reasonable, since gauge transformations will keep one in the same gauge-equivalence class of the potential signifying that the definition of symmetry is applied to gauge invariant specification of the system. If we insisted on the strict invariance of the Hamiltonian, then, on the plane also, there is no full translation symmetry when the potential is chosen in any fixed gauge: the identification of a symmetry will be gauge dependent.

Let us now focus in particular on the translation symmetry along the $y$ direction. Under 
$\theta^{\prime}=\theta, y^{\prime}=y+\ell$, the gauge potential, being a 1-form, transforms as,

$$
A_{i}^{\prime}\left(\theta^{\prime}, y^{\prime}\right)=\frac{\partial x^{j}}{\partial x^{\prime}} A_{j}(\theta, y) \quad \Longleftrightarrow \quad A_{i}^{\prime}(\theta, y)=A_{i}(\theta, y-\ell) .
$$

Applying this to $A=A^{\zeta}$, one sees that

$$
\begin{aligned}
\left(A^{\zeta}\right)^{\prime}(\theta, y) & =A^{\zeta}(\theta, y-\ell) \Longrightarrow \\
{\left[\left(A^{\zeta}\right)^{\prime}\right] } & =\left[A^{\zeta+B R \ell}\right]
\end{aligned}
$$

Thus a translation along the $y$ direction, changes the gauge equivalence class label from $\zeta$ to $\zeta+B R \ell$. It is obvious now that unless $\ell$ is an integer multiple of $\frac{\hbar}{e B R}$, translations along the $y$-direction are not a symmetry of the system.

To summarize: the existence of gauge inequivalent potentials obstructs the continuous y-translation symmetry but within each gauge equivalence class there is a reduced symmetry of discrete y-translations with the step size given by $\frac{\hbar}{e B R}$. The loss of continuous symmetry is attributable to the non-availability of sufficiently many single valued gauge transformation $(\Lambda(\theta, y) \sim \theta$ not allowed) while the survival of discrete $y$-translations is because of the availability of gauge transformations $g(\theta, y) \sim e^{i m \theta}$.

Several remarks are in order.

(1) Notice that arbitrary y-translations change the gauge equivalence classes only for non-zero magnetic field. For the zero magnetic field therefore continuous y-translations are symmetries. In view of the fact that on non-contractible spaces the magnetic field alone does not constitute a complete gauge invariant specification but the holonomies do, it is important to note that the 'free particle' on a cylinder is to be defined by requiring all holonomies to be equal to 1 . This in particular implies that the magnetic field must be zero and the parameter $\zeta$ must also be zero so that the gauge potential is in the equivalence class of the 'zero' gauge potential. In this case of course the full symmetry group of the cylinder will be realized. We will deal only with non-zero $B$.

(2) All of the discussion of the classification of (single valued) gauge potentials and symmetries can be carried out at the purely classical level. There is a point about dimensions to be noted though. Mathematically, a gauge potential comes from a connection on a principal bundle and its holonomies lead to the holonomy equivalence classes. Its dimensions can be chosen to be $\left[A^{\text {mathematical }}\right] \sim L^{-1}$ so that the exponent in the definition of the holonomy is dimensionless. In the mathematical definition of holonomy there is of course no $\frac{e}{c \hbar}$. 
One can see easily that one still gets an equation similar to (37). The subsequent analysis of y-translations leads to the same conclusion about the loss of continuous y-translation symmetry. In this way one can argue that even at the classical level, there is a loss of continuous y-translation symmetry. One will still have the discrete translations as a symmetry, but the step size will not involve any $\hbar$. In conventional (Gaussian for electromagnetism) units, $\frac{e}{c} A^{\text {physical }}$ has dimensions of $M^{1} L^{1} T^{-1}$. To define a holonomy of $A^{\text {physical }}$, definable for arbitrary configurations of sources, one must use a universal constant with dimensions $M^{1} L^{2} T^{-1}$. There is only one such fundamental constant which is $\hbar$. Thus one identifies: $A^{\text {mathematical }}:=\frac{e}{c \hbar} A^{\text {physical }}$. One sees that in order that a physical vector potential exhibits the geometrical properties of a connection on a principal bundle, a quantum framework is essential. The step size thus has a quantum origin.

(3) Note that at the classical level, one could conceivably allow non-single valued gauge potentials (since the potential plays only a local role). This will then also enlarge the class of gauge transformations. With this extended notion of gauge equivalence, one would obtain a single equivalence class of gauge potentials and consequently continuous y-translations would be admissible symmetry transformations. Quantum mechanically, we do not have this option.

From now on we introduce $\mu:=\frac{e B R}{\hbar}=\frac{\tilde{\Phi}}{\Phi_{0}}$ which is the magnetic flux per unit length of the cylinder in units of the flux quantum. The step size for discrete y-translations is then denoted by $\mu^{-1}$. In the light of the remark (1) above, we will also put $\zeta=B R \rho$ for notational convenience and use $\rho$ to label the gauge equivalence classes.

\section{Spectrum, wavefunctions and symmetry realization}

We now proceed to obtain, as in the case of the plane, the spectrum of states, their degeneracies and organization in terms of the symmetry group, $S O(2) \times \mathbb{Z}$. We will also see that the the symmetry group is represented projectively.

Let us choose any one of the gauge equivalence class, labeled by $\rho$, and write the corresponding Hamiltonian in the usual manner. Introducing the notation:

$$
\pi_{y}^{\rho}:=p_{y}-e A_{y}^{\rho} \quad p_{y}:=-i \hbar \partial_{y} \quad, \quad \pi_{\theta}^{\rho}:=p_{\theta}-e A_{\theta}^{\rho} \quad p_{\theta}:=-i \hbar \partial_{\theta}
$$


it follows that

$$
\left[\pi_{\theta}^{\rho}, \pi_{y}^{\rho}\right]=i \hbar e\left(\partial_{\theta} A_{y}^{\rho}-\partial_{y} A_{\theta}^{\rho}\right) \quad=i \hbar e B R
$$

The Hamiltonian is given by,

$$
H^{\rho}=\frac{\left(\pi_{y}^{\rho}\right)^{2}+R^{-2}\left(\pi_{\theta}^{\rho}\right)^{2}}{2 m}=\frac{e B}{2 m}\left(P_{\rho}^{2}+Q_{\rho}^{2}\right), \quad Q_{\rho}:=\frac{\pi_{\theta}^{\rho}}{R \sqrt{e B}}, P_{\rho}:=\frac{\pi_{y}^{\rho}}{\sqrt{e B}}
$$

From these, it follows that the energy eigenvalues are $\hbar \omega\left(N+\frac{1}{2}\right)$ with $\omega:=\frac{e B}{\hbar m}$ and $N$, a non-negative integer. The eigenvalues are manifestly in terms of the magnetic field and are also independent of $\rho$ labeling the gauge equivalence class. The degeneracy of all the eigenvalues is the same and is determined from the degeneracy of the ground state.

The subspace of ground states is obtained by solving $\left(Q_{\rho}+i P_{\rho}\right) \Omega_{\rho}(\theta, y)=0$. The explicit, normalized solutions for the ground states are given by,

$$
\Omega_{\rho, n}(\theta, y)=\frac{1}{\sqrt{2 \pi}}\left(\frac{\mu}{\pi R}\right)^{\frac{1}{4}} e^{i(n+q) \theta} e^{-\frac{\mu}{2 R}\left(y-\rho+\frac{n+q}{\mu}\right)^{2}}, \quad \text { for } n \in \mathbb{Z} .
$$

For a fixed $q$ and $\rho$ one does have infinite degeneracy labeled by $n$, just as in the case of the plane. Can we organize this degeneracy as a representation of the symmetry group $S O(2) \times \mathbb{Z} ?$

To check this we proceed as in the case of the plane. We look for operators of the form

$$
\begin{aligned}
& \Sigma_{\theta}^{\rho}:=\pi_{\theta}^{\rho}+\sigma_{\theta}(\theta, y), \\
& \Sigma_{y}^{\rho}:=\pi_{y}^{\rho}+\sigma_{y}(\theta, y),
\end{aligned}
$$

and require that these commute with the $\pi_{i}^{\rho}$ 's. A quick calculation shows that $\sigma_{\theta}(\theta, y)=$ $-e B R y+C_{\theta}$ and $\sigma_{y}(\theta, y)=e B R \theta+C_{y}$, where $C_{\theta}, C_{y}$ are constants. The functions $\sigma_{i}$ must be single valued to preserve the quasi-periodicity of the wave functions. However $\sigma_{y}$ is not a single valued function on the cylinder and consequently $\Sigma_{y}^{\rho}$ is not well defined as an operator. One thus has only one self adjoint generator $\Sigma_{\theta}^{\rho}=-i \hbar \partial_{\theta}-e B R \rho+C_{\theta}$ commuting with the Hamiltonian and consequently, only a one (continuous) parameter unitary group of symmetries is possible. It generates translations along the $\theta$-direction. Infinitesimal translations along the $y$-direction are not symmetries of the Hamiltonian for any choice of the gauge equivalence class. This manifests the loss of continuous y-translation symmetry.

But although the function $\sigma_{y}(\theta, y)=e B R \theta+C_{y}$ is not well defined on the cylinder, its exponential, $e^{i \frac{a}{\hbar} \sigma_{y}} i s$, provided $a \mu \in \mathbb{Z}$. Therefore, finite translations are admissible and the symmetry group is $S O(2) \times \mathbb{Z}$, possibly projectively realized. 
Define finite translations operators for $\phi \in[0,2 \pi)$ and $a \mu \in \mathbb{Z}$ :

$$
\begin{aligned}
U^{\rho}(\phi) & :=e^{i \frac{\phi}{\hbar} \Sigma_{\theta}^{\rho}}=e^{-i \phi\left(\mu \rho-\frac{C_{\theta}}{\hbar}\right)} e^{\phi \partial_{\theta}} \\
V^{\rho}(a) & :=e^{i \frac{a}{\hbar} \Sigma_{y}^{\rho}}=e^{i a\left(\mu \theta+\frac{C_{y}}{\hbar}\right)} e^{a \partial_{y}}
\end{aligned}
$$

so that

$$
\begin{aligned}
& \left\{U^{\rho}(\phi) \psi\right\}(\theta, y)=e^{-i \phi\left(\mu \rho-\frac{C_{\theta}}{\hbar}\right)} \psi(\theta+\phi, y), \\
& \left\{V^{\rho}(a) \psi\right\}(\theta, y)=e^{i a\left(\mu \theta+\frac{C_{y}}{\hbar}\right)} \psi(\theta, y+a) .
\end{aligned}
$$

It is easily verified that

$$
U^{\rho}(\phi) V^{\rho}(a)=e^{i a \mu \phi} V^{\rho}(a) U^{\rho}(\phi)
$$

and

$$
\begin{aligned}
U^{\rho}(\phi) H^{\rho} & =H^{\rho} U^{\rho}(\phi), \\
V^{\rho}(a) H^{\rho} & =H^{\rho} V^{\rho}(a) .
\end{aligned}
$$

The equation (48) above shows that the symmetry group $S O(2) \times \mathbb{Z}$ is projectively realized, independent of the choice of the constants $C_{\theta}, C_{y}$ and the choice of $\rho$ (compare eq.(25)). The equations (49) are formally valid even without imposition of the condition $a \mu \in \mathbb{Z}$. As stated already in subsection $1 \mathrm{E}$, the Hamiltonian cannot distinguish between the cylinder and its covering plane and so is insensitive to the discretizing condition. The requirement that $V(a)$ respect the quasi-periodicity of the wave functions restricts $a$.

Now the fact that the translations along the $\theta$ directions form the group $S O(2)$, requires that $U^{\rho}(2 \pi)$ is the identity transformation. This together with the quasi-periodicity condition implies that $C_{\theta}=\hbar(\mu \rho-q)$ which fixes the definition of $U^{\rho}(\phi)$ appropriate for quasi-periodicity parameter $q$. There is no such requirement for $V^{\rho}(a)$, so $C_{y}$ is arbitrary and we may take it to be zero for notational convenience.

The action of the group on the states of eq. (44) is obtained as:

$$
\begin{aligned}
& U^{\rho}(\phi) \Omega_{\rho, n}(\theta, y)=e^{-i q \phi} \Omega_{\rho, n}(\theta+\phi, y)=e^{i n \phi} \Omega_{\rho, n}(\theta, y), \\
& V^{\rho}\left(k \mu^{-1}\right) \Omega_{\rho, n}(\theta, y)=e^{i k \theta} \Omega_{\rho, n}\left(\theta, y+k \mu^{-1}\right)=\Omega_{\rho, n+k}(\theta, y) \text {. }
\end{aligned}
$$

The functions $\Omega_{\rho, n}(\theta, y)$ are thus eigenfunctions of action of the $S O(2)$ subgroup of the symmetry group while the action of the $\mathbb{Z}$ subgroup mixes these eigenfunctions. 
One can also construct an eigenbasis such that the $\mathbb{Z}$ subgroup acts diagonally and $S O(2)$ action mixes the basis states. These are essentially obtained by a Fourier transform and are defined as,

$$
\begin{aligned}
\tilde{\Omega}_{\rho, \xi} & :=\sum_{n \in \mathbb{Z}} e^{i n \xi} \Omega_{\rho, n} \quad \forall \xi \in[0,2 \pi) ; \\
V^{\rho}\left(k \mu^{-1}\right) \tilde{\Omega}_{\rho, \xi} & =e^{-i k \xi} \tilde{\Omega}_{\rho, \xi} \\
U^{\rho}(\phi) \tilde{\Omega}_{\rho, \xi} & =\tilde{\Omega}_{\rho, \xi+\phi}
\end{aligned}
$$

We conclude that, as in the case of the plane, the degeneracies are also classified by the

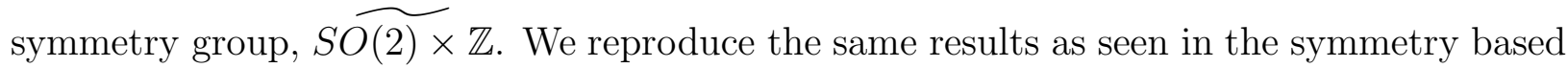
approach of the previous section.

In the present approach, we have two arbitrary parameters: $\rho$, labeling the gauge equivalence classes and $q$, the quasi-periodicity parameter. Only one of them is physically relevant as can be seen from the following argument.

While the energy eigenvalues are independent of $\rho$ and $q$, the wave functions are not. In particular, for example, $\left|\Omega_{\rho, 0}\right|^{2}(\theta, y)$ is a Gaussian in $y$, centred at $y=\rho-q \mu^{-1}$. So $\rho$ and $q$ together, in this particular combination, determine the peak of the Gaussian along the $y$-axis. Since the choice of the origin along the $y$-axis is arbitrary, we can always choose it to coincide with this peak. Equivalently, given a quasi-periodicity parameter $q$, we can always choose the gauge equivalence class labeled by $\rho=q \mu^{-1}$ without any loss of generality. Having made this choice, we are left with only the quasi-periodicity parameter $q$ exactly as in the previous section. Notice that $\rho \rightarrow \rho+\mu^{-1}$ corresponds precisely to $q \rightarrow q+1$ and $q$ is defined modulo integers. The $\rho$ is thus seen as parameterizing different, unitarily equivalent ways of writing the wavefunction UR corresponding to different choices of origin along the $y$-axis.

Incidentally, by identifying $R \theta$ with $x$ and relaxing the identification of $\theta=0$ and $\theta=2 \pi$, reproduces the planar case as expected.

\section{SUMMARY AND DISCUSSION}

We summarize our results and conclusions in the form of a series of remarks.

(1) In the symmetry approach, reduction of the continuous translational symmetry group to a discrete subgroup is a result of the non-existence of PURs for the full symmetry group 
of the cylinder. In the Hamiltonian approach, the same result is seen to be a consequence of the structure of the group of gauge transformations and the corresponding existence of gauge inequivalent potentials. In both approaches, the basic cause of the phenomenon is the non-contractible nature of the configuration space.

(2) It is interesting to note that as $\hbar \rightarrow 0$ and/or $R \rightarrow \infty$, the basic translation step, $\mu^{-1}=\frac{\hbar}{e B R}$, goes to zero implying essentially continuous translations being restored. The large $R$ limit takes us towards the planar approximation while the vanishing $\hbar$ limit takes us to the classical picture where continuous y-translations symmetry should be and is recovered. This is understandable in view of the remark (3) in IIC] The step size is relevant only for non-zero magnetic field and the 'free particle' limit is to be understood ab initio as discussed in the remark (1) in $11 \mathrm{C}$

(3) It would be very interesting to experimentally verify the discretization of the translation symmetry implied by quantization. Restoring the conventional units $\left(c \sim 3 \times 10^{10}\right.$ $\mathrm{cm} / \mathrm{sec}$ ), the basic translation step selected by the system is $a=\mu^{-1}=\frac{\hbar c}{e B R} \sim$ $6.6 \times 10^{-8} B^{-1} R^{-1}$ centimeters. This is extremely small eg. for $R \sim 1 \mathrm{~cm}$ and $B \sim 1$ gauss, the basic step is only of the order of an angstrom. Needless to say that some very clever experimental tricks would be needed to verify the discretization.

(4) Similar conclusions hold in the case of the torus. The torus group $S O(2) \times S O(2)$ has no non-trivial central extensions. Its subgroups of the form $\mathbb{Z}_{m} \times \mathbb{Z}_{n}$ where $m, n$ are positive integers do have central extensions and they are represented unitarily on the state space [5]. Intuitively one expects the torus problem to effectively model a finite sample system. Indeed in a recent paper 8] discussing the finite geometry problem relevant for the quantum Hall effect, this expectation has been corroborated to an extent. One may likewise expect our cylinder results to model the problem on a strip.

(5) To summarize: At the level of a physical prediction, we see that for the cylinder Landau electron, there is a spontaneous "crystallization" with lattice spacing along the axial direction being determined by the radius and the magnetic field. At the theoretical level, an important lesson is that symmetries indicated by the classical magnetic field are not necessarily the ones realized quantum mechanically. One needs to pay attention to a complete gauge invariant specification of the system and then discover the allowed symmetries. The symmetry based approach, which is manifestly gauge invariant, automatically gets the realizable symmetries directly by using only the general principles of symmetry realization 
in a quantum framework.

\section{Acknowledgments}

PPD expresses his appreciation to the Tata Institute of Fundamental Research, Mumbai for hospitality. Both of us acknowledge discussions with Prof. M. Barma and Prof. G. Baskaran.

[1] E. P. Wigner, Group Theory and its Applications to the Quantum Mechanics of Atomic Spectra, Academic Press, New York, 1959.

[2] For a sample of different approaches to quantization in general, see for example, C. J. Isham, in Relativity, Groups and Topology II, Ed. B. S. DeWitt and R. Stora, NorthHolland, Amsterdam, 1984;

N. M. J. Woodhouse, Geometric Quantization, second edition, Clarendon, Oxford, 1992;

Abhay Ashtekar, Jerzy Lewandowski, Donald Marolf, Jose Mourao, Thomas Thiemann, Jour. Math. Phys., 36, 6456, 1995;

[3] P. P. Divakaran, Rev. Math. Phys., 6, 167, 1994;

P. P. Divakaran, Phys. Rev. Lett., 79, 2159, 1997;

[4] M. S. Raghunathan, Rev. Math. Phys., 6, 207, 1994.

[5] P. P. Divakaran and A. K. Rajagopal, Int. J. Mod. Phys., 9, 261, 1995.

[6] D. Mumford, M. Nori and P. Norman, Tata Lectures on Theta III, Birkhäuser, 1991, Chap 1; P. P. Divakaran, preprint, e-print archive math-ph/9904004.

[7] T. T. Wu and C. N. Yang, Phys. Rev., D 12, 3845, 1976.

[8] F. Chandelier, Y. Georgelin, T. Masson, J.-C. Wallet, Ann. Phys., 305, 79, 2003.

For an earlier discussion of the torus problem in an algebraic quantization scheme see, V. Aldaya, M. Calixto, J. Guerrero, Comm. Math. Phys., 178, 399, 1996. 